\title{
Valorization of bio-waste material: future dimensions for path towards sustainability
}

\author{
Richa Kothari ${ }^{1} \cdot$ Anita Singh $^{1}$ - A. K. Pandey ${ }^{2}$. V. V. Tyagi ${ }^{3}$. Dilfuza Egamberdieva ${ }^{4,5}$. \\ Sonoko D. Bellingrath-Kimura ${ }^{5,6} \cdot$ Naveen Kumar Arora $^{7}$
}

Published online: 31 May 2021

๑) Society for Environmental Sustainability 2021

Management of large amounts of wastes present in the surrounding environments of humans and in natural ecosystems is a challenging issue. Waste management is one of the major issues for the humankind, and will be the part of future challenges in coming years due to rapid increase in population and sharp decrease of natural resources. Thus, $3 \mathrm{R}$ principles of reduce, reuse and recycle are the important triangle for the conversion of waste into valuable resources to improve the development strategies in a sustainable way and give impetus to circular economy. But, now-a-days with the help of research and technological advancements, waste can be converted into useful products which will not only lead to environmental sustainability but also boost the economy. Biodegradable and non-biodegradable wastes are generated in huge amounts throughout the world from wide spectrum of sources including domestic, industrial, and agricultural activities. The waste materials have a good share of

Richa Kothari

kothariricha21@gmail.com; richakothari786@gmail.com

1 Department of Environmental Sciences, Central University of Jammu, Rahya-Suchani, Bagla, Samba, Jammu 181143, $\mathrm{J} \& \mathrm{~K}$, India

2 Research Centre for Nano-Materials and Energy Technology (RCNMET), School of Engineering and Technology, Sunway University, No. 5, Jalan Universiti, Bandar Sunway, 47500 Petaling Jaya, Selangor, Malaysia

3 School for Energy Management, Shri Mata Vaishno Devi University, Kakryal, Katra 182320, J\&K, India

4 Faculty of Biology, National University of Uzbekistan, Tashkent, Uzbekistan 100174

5 Leibniz Centre for Agricultural Landscape Research (ZALF), Müncheberg, Germany

6 Faculty of Life Sciences, Humboldt University of Berlin, Berlin, Germany

7 Department of Environmental Science, School of Earth and Environmental Sciences, BBA University, Lucknow, UP, India biodegradable organic carbon which can be converted into valuable products. The vast generation, pervasive presence and chemical richness of these wastes make their valorization as one of the most promising way for reducing environmental impacts realizing the concept of waste to wealth. The conversion of waste into wealth includes reuse and recycling of resources which reduce insecurity of resource supply, the cost of waste disposal, environmental burden, and result in sustainable utilization of feedstock. The use of side products is an innovative and economically sustainable approach which generates additional revenues. The bio-wastes include- forestry residues, agricultural residues, animal wastes, industrial bio-wastes, cattle manure, fly ash, bio-sludge, municipal solid bio-wastes, and algal biomass, generated in huge amount every year globally, grabbing the worldwide attention for its management. These underutilized bio-wastes can be converted into value-added products by using different strategies for their processing. Valorization of bio-waste materials through various processing routes existed since long but cost-effectiveness along with fulfilling the sustainable need is somehow showing gaps and challenges as per literature available. This special issue show cases some promising strategies with efficient waste management protocols and research initiatives in the form of research and review articles.

The bio-waste feedstock can be converted into valuable products via chemical, thermo-chemical or biological pathways which provide sustainable solutions for environmental management. Solar energy which is prime source of any form of energy can also be efficiently utilized in bio-waste valorization. If the pyrolysis heat process is supplied from a concentrated solar system, which can be achieved at reasonably moderate solar radiations, it can improve the efficiency reasonably well as compared to conventional processes. Microorganisms play an important role in conversion of feedstock into end product even by utilizing harsh chemical wastes in conversion process. The optimization of process 
parameters is an important and essential step in increasing the efficiency of process of conversion of bio-waste into environmentally sustainable value added products. Therefore, keeping all the applications for available bio-waste in a sustainable manner this special issue is framed with the title "Bio-waste valorization for environment sustainability". A large focus needs to be put on valorization of bio-waste for sustainable economy with clean environment with approach of waste minimization too. Hence, the special issue was planned with following objectives; (1) low-cost and environmentally sustainable feedstocks; (2) pretreatment of bio-waste materials; (3) pathways for improved and efficient biosystem; (4) role of microbes in integration with bio-waste materials utilization; (5) optimization of process parameters and organic waste utilization for value-added product; (6) conversion of waste for energy options through solar energy. This special issue is focused towards bio-waste materials like agro-industry waste (leaf waste, fruit peels, vegetable waste), food waste, microorganisms (dead and alive), flower waste, municipal organic waste, chicken feathers etc. and for wastewater treatment, heavy metal removal and value-added products (bio-energy options). Researchers from national as well as international platforms discussed the long-term solutions with practical approaches to reduce the use of fossil-fuel and their emissions. Furthermore, advanced technologies like nanoparticles tailored lignocellulosic biochar developed from agri-wastes for the remediation of heavy metals like cadmium (II) and chromium (VI) from aqueous system and utilization of nanomaterials for scaleup of bioenergy options (liquid biofuels) related research work are also included. Innovative uses of biochar such as for remediation of degraded and polluted agri-ecosystems, and biocontrol of phytopathogens are coming up. The novel aspects of formulating and utilizing biochar for attaining the targets of agricultural sustainability are explained.

Production of high-value products from bio-waste material via biotechnological approaches (e.g., solid state fermentation) and with enzymatic reactions, depends on the type of residual compounds and requires continuous research for optimization and increasing the efficiency. Similar type of findings with experimental plans and investigations are also included with the concept of maximizing the use of resources, minimization of waste generation and optimisation of the process. Types of bio-wastes with their composition, technical aspects, and barriers in utilization, degradation and bioconversion, have significance for improving the liquid as well as solid waste treatments for practical applications. Pretreatment options for utilization of bio-waste to make it more viable for further utilization is also an important aspect for research and for obtaining desirable products from the waste. Importance of gasification technologies in comparison to coal based thermal power plants, offering the potential for clean and efficient energy on the basis of simulated results obtained with co-gasification as better option over other conventional systems is another important aspect covered in this issue. Role of microbial leaching in heavy metal treatment options with bio-waste materials is also researched upon. The work presented in the special issue will also help in opening up future directions of research on diverse aspects of utilization of biowaste, and utilize it in different key sectors such as sustainable agriculture, clean and green energy, bio based industries including enzymes, pharmaceuticals, bioplastics etc.

All the editor(s) are hopeful that this special issue will help to describe and resolve the challenges involving utilization of bio-waste material for conversion to value-added products. The research work presented will also be useful in reducing the waste material and make the planet a better place to inhabit for diverse life forms. A sustainable and successful strategy for handling and utilization of biowaste requires an integrated approach to recover the maximum environmental and economic values from the different types of wastes already present or produced due to anthropogenic activities.

Publisher's Note Springer Nature remains neutral with regard to jurisdictional claims in published maps and institutional affiliations. 